\title{
Genome variation in colorectal cancer patient with liver metastasis measured by whole-exome sequencing
}

\author{
Hui Yi ${ }^{1,2} \wedge$, Zhi-Wei Liao ${ }^{2}$, Jun-Jie Chen ${ }^{1}$, Xin-Yu Shi ${ }^{1}$, Guo-Liang Chen ${ }^{1}$, Guan-Ting Wu ${ }^{1}$, \\ Di-Yuan Zhou ${ }^{1}$, Guo-Qiang Zhou ${ }^{5}$, Jin-Yu Huang ${ }^{6}$, Lian Lian ${ }^{4}$, Zheng-Yuan Yu $^{3}$, Song-Bing $\mathrm{He}^{1}$ \\ ${ }^{1}$ Department of General Surgery, The First Affiliated Hospital of Soochow University, Suzhou, China; ${ }^{2}$ Department of General Surgery, Renhe \\ Hospital, Baoshan District, Shanghai, China; ${ }^{3}$ Department of Oncology, First Affiliated Hospital of Soochow University, Suzhou, China; \\ ${ }^{4}$ Department of Oncology, Suzhou Xiangcheng People's Hospital, Suzhou, China; ${ }^{5}$ Department of Gastrointestinal Surgery, Changshu No. 2 \\ Hospital, Suzhou, China; ${ }^{6}$ Department of Radiology, the First Affiliated Hospital of Soochow University, Suzhou, China \\ Contributions: (I) Conception and design: SB He, ZY Yu, H Yi; (II) Administrative support: SB He, ZY Yu; (III) Provision of study materials or \\ patients: SB He, L Lian, GQ Zhou, DY Zhou, GT Wu, ZW Liao; (IV) Collection and assembly of data: H Yi, JJ Chen, XY Shi, GL Chen, ZY Yu; (V) \\ Data analysis and interpretation: H Yi, ZY Yu, SB He; (VI) Manuscript writing: All authors; (VII) Final approval of manuscript: All authors. \\ Correspondence to: Song-Bing He. Department of General Surgery, The First Affiliated Hospital of Soochow University, Suzhou, China. \\ Email: hesongbing1979@suda.edu.cn; Zheng-Yuan Yu. Department of Oncology, First Affiliated Hospital of Soochow University, Suzhou, \\ China. Email: strongeryy1985@163.com; Lian Lian. Department of Oncology, Suzhou Xiangcheng People’s Hospital, Suzhou, China. \\ Email: dr_lianlian@163.com.
}

Background: Liver metastasis of colorectal cancer (CRC) is an important cause of death from CRC, but its molecular mechanism is still unclear. In recent years, whole-exome sequencing has played an increasingly important role in the study of the occurrence and development of diseases, especially malignant tumors. Its high throughput and low cost advantages enable researchers to explore the pathogenic genes of diseases, and screen potential molecular markers and therapeutic targets from the level of genomics.

Methods: This study collected the primary tumor tissues, matched paracancerous, normal tissues, and liver metastases of 4 CRC patients admitted to the Department of General Surgery of the First Affiliated Hospital of Soochow University, and performed high-depth whole-exome sequencing, with the sequencing depth of each sample reaching $123 \times$ on average, then filtered the sequencing data, compared them, and analyzed the bioinformatics data.

Results: we found 8,565 single nucleotide variants (SNV) and 429 insertions/deletions (InDel) in the primary and hepatic lesion tissues, and the genes with the highest mutation frequency were titin (TTN), obscurin (OBSCN), and homeodomain-interacting protein kinase 2 (HIPK2). The Gene Ontology (GO) and Kyoto Encyclopedia of Genes and Genomes (KEGG) pathway analysis of the mutant genes was conducted, and it was found that the mutant genes were mainly concentrated in the cells, cell parts, and cellular process of GO. The results of KEGG pathway analysis showed that mutations were mainly distributed in circadian entrainment, insulin secretion, and glutamatergic synapse. Further, we identified 723 SNV and Indel genes with high frequency mutations including TTN, OBSCN, and hydrocephalus-inducing protein homolog (HYDIN) across all tissues of liver metastases. The GO analysis showed that the mutated genes in liver metastatic tissues were mainly concentrated in cell, cell part, and cellular process. The KEGG pathway analysis showed that high frequency mutation genes were focused on gastric acid secretion, bile secretion, and melanogenesis.

Conclusions: This study found some candidate genes related to the occurrence of CRC and liver metastasis through whole-exome sequencing of relevant tissues in CRC patients with liver metastasis, which is expected to provide new markers and therapeutic targets for such patients.

\footnotetext{
^ ORCID: 0000-0001-5894-2488.
} 
Keywords: Whole-exome sequencing; colorectal cancer (CRC); liver metastasis

Submitted Dec 10, 2020. Accepted for publication Feb 08, 2021.

doi: 10.21037/jgo-21-9

View this article at: http://dx.doi.org/10.21037/jgo-21-9

\section{Introduction}

Colorectal cancer (CRC) is one of the most common digestive system malignancies in the world. In China, nearly one fifth of CRC patients have been found to have liver metastasis (LM) at their first diagnosis, and half of CRC patients have LM during the disease progression. The involvement of LM is a very important factor in the prognosis of CRC $(1,2)$, it is caused by the interaction of multiple factors, and its clinical phenotype, characteristics, and molecular mechanism of metastasis are also currently hot topics in both clinical and basic research. The variation of tumor cell genome is an important factor for the occurrence and metastasis of tumors (3-5). In recent years, whole-exome sequencing has been widely used in the study of various diseases, especially in oncology research. Several functional genes associated with lung cancer targeting or immunotherapy have been identified in non-small cell lung carcinoma (NSCLC) (6,7). It has also been repeatedly confirmed by whole-exome sequencing that protein 53 (P53), Kirsten rat sarcoma viral oncogene homolog (KRAS), adenomatous polyposis coli (APC), and other genes play an important role in the occurrence and progression of CRC $(8,9)$. However, the genomic characteristics of LM from CRC are still unclear. Though there are many studies on the NGS of colorectal cancer, but relatively few studies on the gene detection of colorectal cancer with liver metastasis, and the data of exome sequencing of colorectal cancer with liver metastasis are even less in China.

In this study, primary tumor tissues, matched normal tissues, and liver metastasis specimens of 4 CRC patients with LM from the General Surgery Department of the First Affiliated Hospital of Soochow University were collected, and high-depth complete exome sequencing was performed to analyze the mutated genes, tumor mutation burden (TMB), molecular functions, and signal pathways related to CRC with LM. It is hoped that this study can deepen the understanding of CRC with LM and provide potential marker molecules and therapeutic targets for the diagnosis and treatment of CRC with LM. We present the following article in accordance with the MDAR reporting checklist (available at http://dx.doi.org/10.21037/jgo-21-9).

\section{Methods}

\section{Participants and samples}

\section{CRC patient sample acquisition}

We collected primary CRC tumor tissues, matched normal tissues, and liver metastatic tumor tissues of 4 patients from the Department of General Surgery of the First Affiliated Hospital of Soochow University from September 2019 to April 2020. Informed written consent was provided by all patients before inclusion in this study. Respective tumor tissue samples from a histologically confirmed adenocarcinoma by two molecular pathologists were matched with the inclusion criteria. This study was conducted in accordance with the Declaration of Helsinki (as revised in 2013) and was approved by the Ethics Committee of the First Affiliated Hospital of Soochow University (No. 2021-023).

\section{DNA extraction}

The DNA was extracted from primary tumor tissues, liver metastatic tissues, and matched normal tissues using E.Z.N.A. Tissue DNA kit (Omega Bio-Tek, Norcross, GA, USA) and QIAamp DNA FFPE Tissue kit (Qiagen Sciences, Venlo, Netherlands). The DNA extracted from normal tissues were used as germline DNA control. The samples with cancer cell populations were estimated by pathologists to ensure more than $75 \%$ of cells were cancer cells. A bioanalyzer (Agilent, Palo Alto, CA, USA) was used to assess DNA quantity.

\section{Whole-exome sequencing and data processing}

Sample genome DNA was fragmented using NEBNext dsDNA Fragmentase (NEB, Ipswich, MA, USA) followed by DNA ends repairing. End-repaired DNA fragments were detailed and ligated with the NEBNext adaptor (NEB, Ipswich, MA, USA). Biotinylated RNA library baits and magnetic beads were mixed with the barcoded library for selection of targeted regions using the SureSelect Human All Exon V6 Kit (Agilent Technologies, Palo Alto, CA, USA). The captured sequences were further amplified for $150 \mathrm{bp}$ paired-end sequencing in Illumina $\mathrm{X}$-ten system (Illumina, San Diego, CA, USA). 
Table 1 Clinicopathological participant characteristics

\begin{tabular}{|c|c|}
\hline Characteristics & No. of cases [\%] \\
\hline \multicolumn{2}{|l|}{ Total number } \\
\hline Age, years, mean [range] & $64.5[46-77]$ \\
\hline \multicolumn{2}{|l|}{ Gender } \\
\hline Male & $3[75]$ \\
\hline Female & $1[25]$ \\
\hline \multicolumn{2}{|l|}{ Pathological diagnosis } \\
\hline Adenocarcinoma & 4 [100] \\
\hline \multicolumn{2}{|l|}{ Tumor stage at diagnosis } \\
\hline III & 0 \\
\hline IV & $4[100]$ \\
\hline \multicolumn{2}{|l|}{ Histological grade } \\
\hline Grade 2 & $2[50]$ \\
\hline Grade 3 & $2[50]$ \\
\hline \multicolumn{2}{|l|}{ Vascular invasion } \\
\hline Yes & $2[50]$ \\
\hline No & $2[50]$ \\
\hline \multicolumn{2}{|l|}{ Lymphatic invasion } \\
\hline Yes & $4[100]$ \\
\hline No & 0 \\
\hline \multicolumn{2}{|l|}{ Perineural invasion } \\
\hline Yes & $2[50]$ \\
\hline No & $2[50]$ \\
\hline \multicolumn{2}{|l|}{ CEA value at diagnosis } \\
\hline Normal & 0 \\
\hline Increased & $4[100]$ \\
\hline
\end{tabular}

\section{Somatic single nucleotide variant and insertion/deletion identification}

To identify somatic single nucleotide variants (SNVs) and insertion/deletions (InDels), the Burrows-Wheeler Aligner (BWA) (10) was used to align the clean reads from each sample against the human reference genome (GRCh38). The picard tool (http://picard.sourceforge.net/index. shtml) removed the read duplicates derived from library polymerase chain reaction (PCR). Somatic SNV and InDel calling was processed for multi-sample by MuTect (https:// software.broadinstitute.org/cancer/cga/mutect) (11). Tumor mutation burden (TMB) is calculated as the number of somatic mutations per million bases (MB), including somatic single nucleotide variants (SNVs) and insertion/ deletions (InDels).

\section{SNV}

The gene sets screened were used for functional annotation analysis using in-house script, which consisted of the Kyoto Encyclopedia of Genes and Genomes (KEGG) (12) and Gene Ontology (GO) (13) database. The significance of gene group enrichment was defined by a modified Fisher's exact test and a $\mathrm{P}$ value $<0.05$ was considered to indicate a statistically significant difference.

\section{Statistical analyses}

The software SPSS (IBM Corp., Armonk, NY, USA) (14) was used to analyze all the correlated biological and clinical variables.

\section{Results}

\section{Participant characteristics}

The mean age of the 4 CRC patients with LM was 64.5 years (range, 46-77 years), and included 3 males and 1 female. All 4 were diagnosed with adenocarcinoma, and did not receive adjuvant chemoradiotherapy before Surgery. The tumor stage of 4 participants were stage IV. Half $(n=2)$ of them were histological grade 2 and another 2 were histological grade 3 . All 4 participants had lymphatic invasion and perineural invasion. The carcinoembryonic antigen (CEA) was increased in all 4 participants (Table 1).

\section{Whole-exome sequencing}

We collected the primary tumor tissues, matched paracancerous normal tissues, and LMs of 4 patients admitted to the Department of General Surgery of the First Affiliated Hospital of Soochow University, and performed high-depth whole-exome sequencing, with the sequencing depth of each sample reaching $123 \times$ on average (Table S1).

\section{Identification of somatic single nucleotide polymorphisms and InDels}

The normal tissue samples data of all participants matched the original site of cancer tissue samples data. To compare the LMs sample data analysis, we took the first participants' normal tissue and named it CRC01C (CRCO1 CONTROL), the original cancer organization 


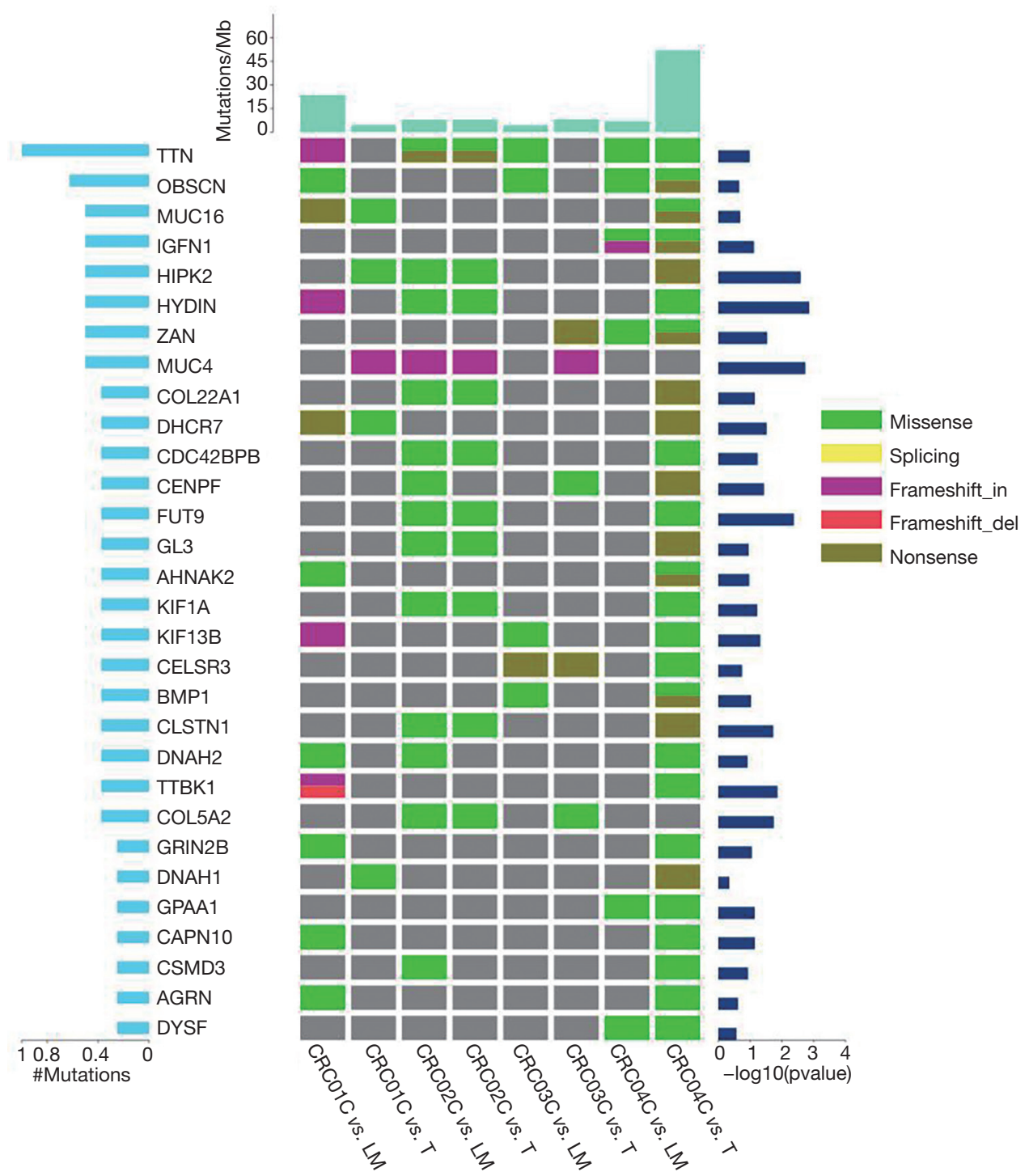

Figure 1 Landscape of somatic mutations in all samples. Figure left: proportion of the number of gene mutations. Figure right: significance of high-frequency mutations. Up: Sample mutation frequency. The types of mutations are shown, including (I) non-synonymous mutations (green), (II) shear site mutations (yellow), (III) causes code shift insertion (purple), and (IV) causes code shift deletion (red).

was named CRC01CvsT (CRCO1 CONTROL vs. tumor), LMs sample was named CRC01CvsLM (CRCO1 CONTROL $v s$. liver metastasis), and then named the rest of the participant samples in the same way. By filtering the sequencing comparing and analyzing the data, 8,565 SNVs and 429 Indels were detected across all samples; CRC04CvsT had the largest number of SNVs $(5,940)$, followed by CRC01CvsLM $(1,094)$ (Table S2). Significantly mutated genes (SMG) are those genes whose mutation frequency is significantly higher than those in the background. Generally, differences of somatic SNV and InDel are analyzed comprehensively. MuSiC (The Genome
Institute, Washington University, USA) software (15) was used to search for genes with higher mutation frequency in tumor samples (compared with control samples). A significantly mutated gene (SMG) test was performed for each type of mutation, whose test method was convolution test (CT). We found that the genes with the highest frequency of mutation were titin (TTN), obscurin (OBSCN), and homeodomain-interacting protein kinase 2 (HIPK2). We identified the 30 genes with the highest frequency of mutations. We also calculated the TMB of each sample respectively on the average TMB over 16 mutations per MB (5-60 mutations/MB) (Figure 1). 


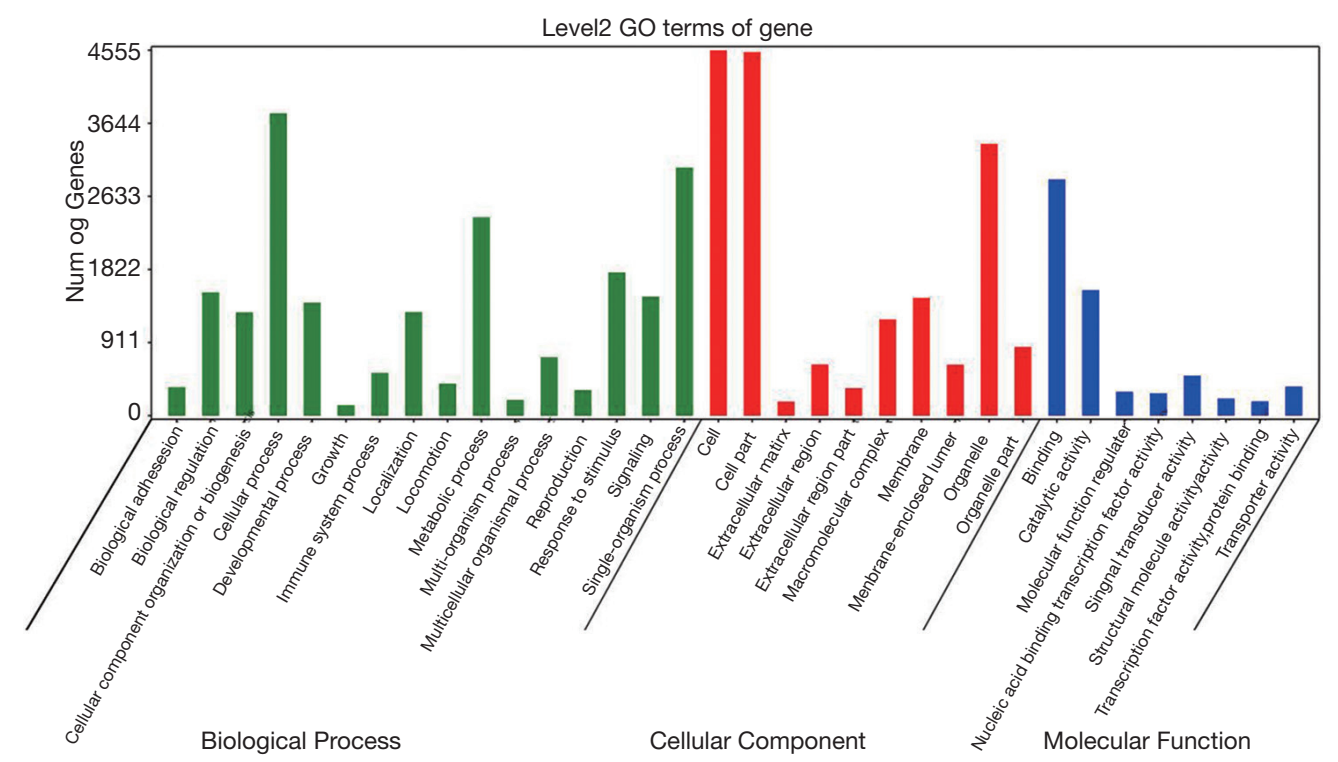

Figure 2 GO analysis histogram of the mutated genes of all samples. GO, Gene Ontology.

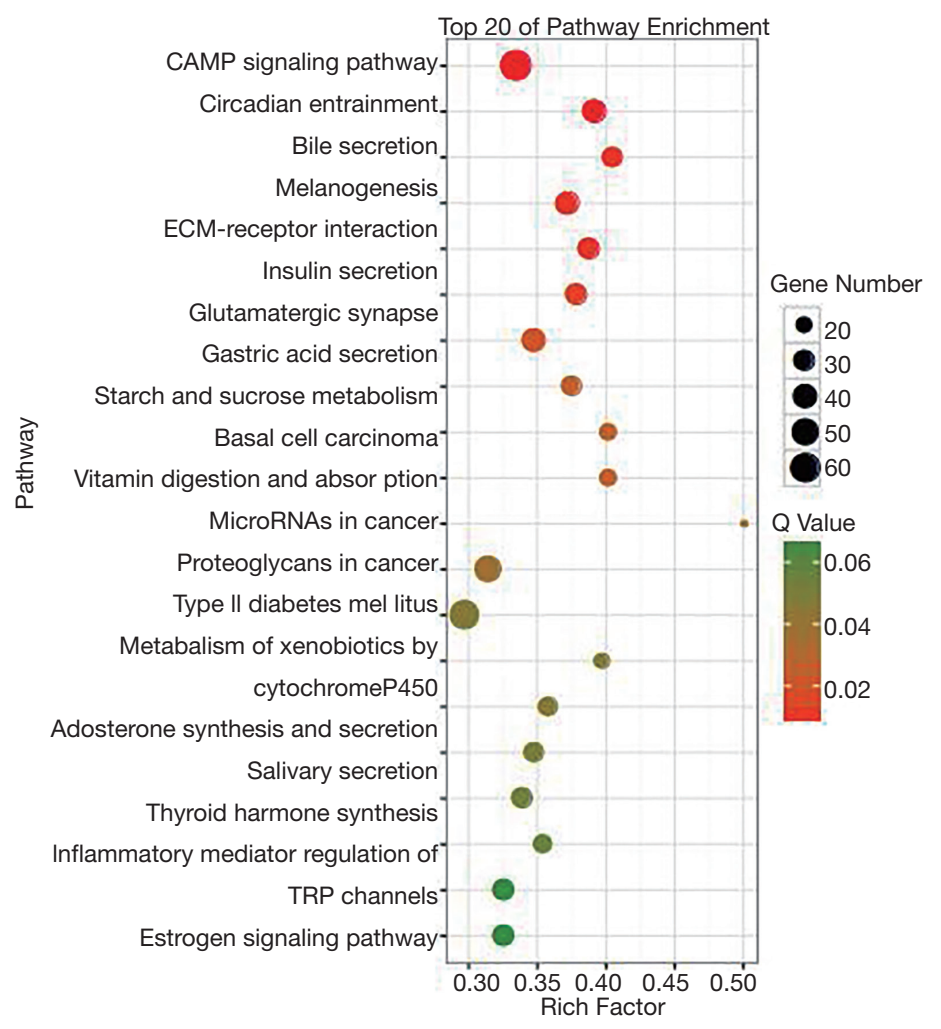

Figure 3 KEGG bubble of mutant genes of all samples. KEGG, Kyoto Encyclopedia of Genes and Genomes.

\section{KEGG and GO analysis}

We conducted GO and KEGG pathway analysis of the mutant genes, revealing that the mutant genes were mainly concentrated in the cells, cell parts, and cellular process of GO (Figure 2). Results of KEGG pathway analysis showed that mutations were mainly distributed in circadian entrainment, insulin secretion, and glutamatergic synapse (Figure 3). 


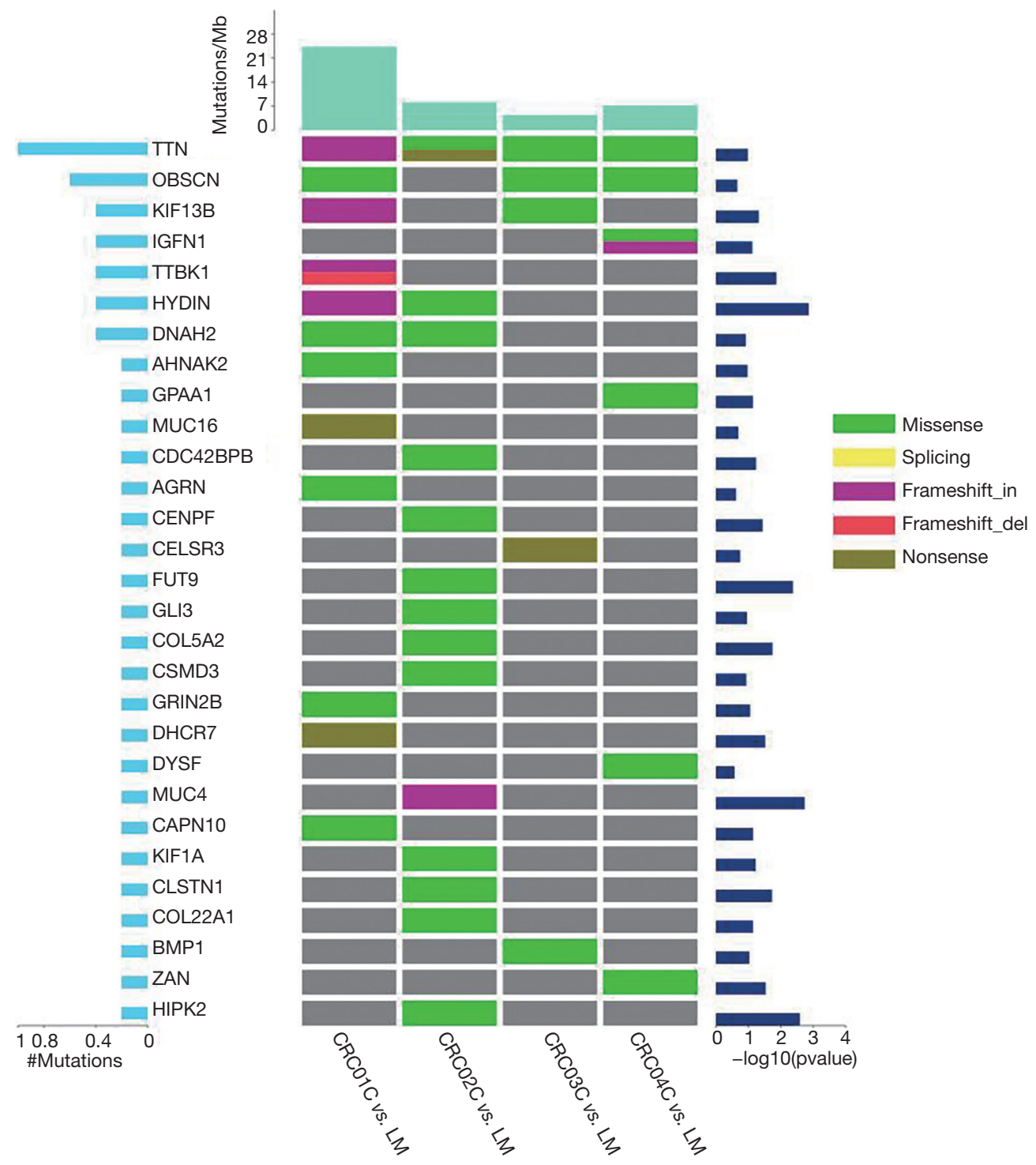

Figure 4 Landscape of somatic mutations in CRCLM samples. Figure left: proportion of gene mutations. Figure right: significance of highfrequencymutations. Figure right: sample mutation frequency. The types of mutations are shown, including (I) non-synonymous mutations (green), (II) shear site mutations (yellow), (III) causes code shift insertion (purple), and (IV) causes code shift deletion (red). CVRCLM, colorectal cancer liver metastasis.

\section{Gene mutation and KEGG and GO analysis in LM samples}

We further analyzed the high frequency mutated genes of LMs, which were TTN, OBSCN and hydrocephalusinducing protein homolog (HYDIN) (Figure 4). The GO analysis showed that the mutated genes in LM tissues were mainly concentrated in the cell, cell parts, and cellular process (Figure 5). The KEGG pathway analysis showed that high frequency mutation genes were focused on gastric acid secretion, bile secretion, and melanogenesis (Figure 6).

\section{Discussion}

Tumor metastasis is an important factor in tumor progression, and distant metastasis is often the main reason for tumor treatment failure. The process of LM of CRC is extremely complex, involving many tumor-related molecules, which are induced and regulated by their respective specific signaling pathways. With the continuous progress of all kinds of research techniques in recent years, a growing body of evidence has shown that tumor development 


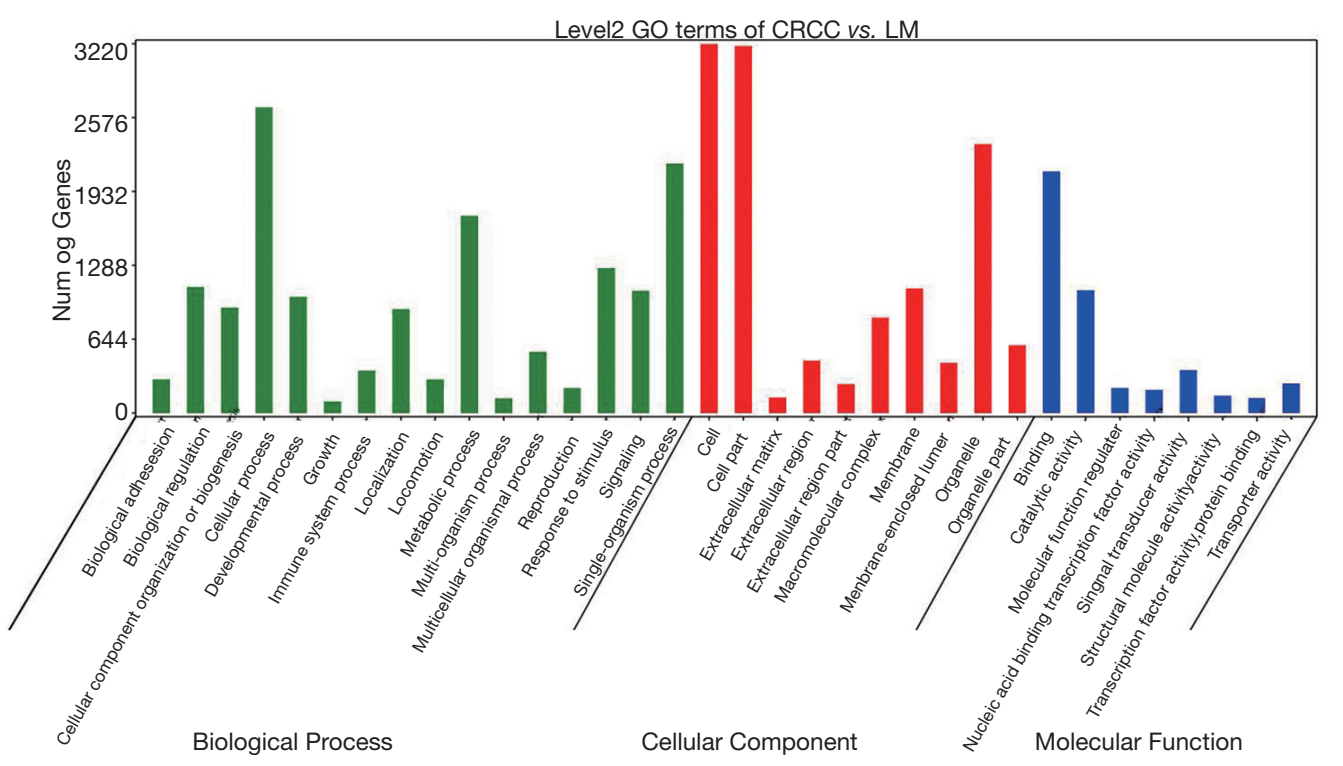

Figure 5 GO analysis histogram of mutant genes in colorectal cancer liver metastasis samples. GO, Gene Ontology.

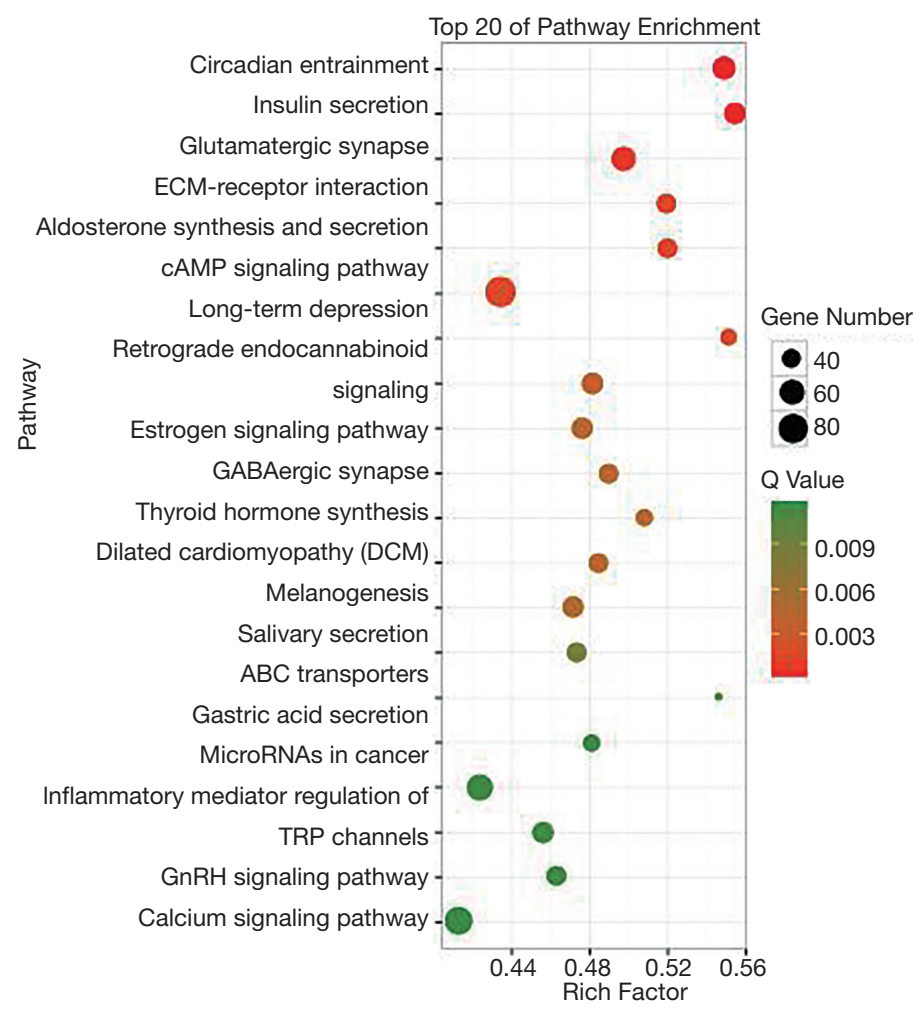

Figure 6 KEGG bubble diagram of liver metastases from colorectal cancer. KEGG, Kyoto Encyclopedia of Genes and Genomes.

and metastasis are related to the cancer genome (16), transcriptome (17), epigenetics (18), proteome (19), metabolome (20), and tumor microenvironment (21) interaction cross-regulation of a dynamic complex process.
This study collected the primary tumor tissues, matched paracancerous normal tissues, and metastatic tissues of 4 patients admitted to the General Surgery Department of the First Affiliated Hospital of Soochow University, 
and conducted high-depth whole-exome sequencing, in an attempt to explore the molecular mechanism of CRC LM from the genomic level. We performed an average sequencing depth of $123 \times$ per sample. By filtering, comparing, and analyzing the data, we identified 8,565 SNVs and 429 Indels in primary and hepatic metastases, and we found that the genes with the highest frequency of mutation were TTN, OBSCN, HIPK2, and HYDIN. The TTN gene was the most frequently mutated gene and had mutations in all 4 participants with LM. The TTN gene is known to encode rhabdomyin, and mutations in this gene are also associated with familial hypertrophic cardiomyopathy (HCM) 9 (22). It has also been identified as a chromosomal structural protein, and TTN has been found to promote bone metastasis in breast cancer (23). We speculated that variation of TTN might lead to chromosome instability and thus promote the occurrence and metastasis of tumors. The OBSCN gene is over $150 \mathrm{~kb}$ long and contains over 80 exons encoding a protein of approximately $720 \mathrm{kDa}$ that belongs to the sarcomeric signaling protein family $(24,25)$. It is an important signaling protein that is involved in the modulation of multiple cellular signals. We hypothesized that OBSCN mutation might lead to abnormal intracellular signaling pathway transduction that catalyzed changes in cell function.

Due to the small number of patients with liver metastasis of colorectal cancer, it is difficult to obtain appropriate tissue samples of liver metastasis of colorectal cancer for sequencing. We will also increase the number of samples of liver metastasis of colorectal cancer for total exon sequencing in the future work to obtain more data. And due to financial constraints, this study failed to combine with other omics studies such as transcriptome and proteomics. We will try to address these issues in the future to further explore the molecular mechanism of CRC LM.

\section{Conclusions}

In this study, we found some candidate genes related to the occurrence of CRC and LM through whole-exome sequencing of relevant tissues in CRC patients with LM. These findings are expected to provide us with new markers and therapeutic targets for CRC patients with LM.

\section{Acknowledgments}

Funding: This work was supported by Project of Nature Science Foundation of China $(81672348,81702048)$,
National Science Foundation of Jiangsu Province of China (BK20191172), Program of Postgraduate Research Innovation in University of Jiangsu Province (KYCX19_1993), Project of Key laboratory of Clinical pharmacy of Jiangsu Province of China (XZSYSKF2020027), Project of Science and Technology Development Plan of Suzhou City of China (SYS2019007), Project of Gusu Medical Key Talent of Suzhou City of China (GSWS2020005), Project of Medical Science and Technology Development Foundation of Suzhou City of China (SYS201734), The Science and Technology Project Foundation of Suzhou (SS201852, SS202093), the Science and Education for Health Foundation of Suzhou for Youth (KJXW2019074) and Project of Medical Specialty and Community of Baoshan District of Shanghai City of China (BSZK-2018-A08).

\section{Footnote}

Reporting Checklist: The authors have completed the MDAR checklist. Available at http://dx.doi.org/10.21037/jgo-21-9

Data Sharing Statement: Available at http://dx.doi. org/10.21037/jgo-21-9

Conflicts of Interest: All authors have completed the ICMJE uniform disclosure form (available at http://dx.doi. org/10.21037/jgo-21-9). The authors have no conflicts of interest to declare.

Ethical Statement: The authors are accountable for all aspects of the work in ensuring that questions related to the accuracy or integrity of any part of the work are appropriately investigated and resolved. This study was conducted in accordance with the Declaration of Helsinki (as revised in 2013) and was approved by the Ethics Committee of the First Affiliated Hospital of Soochow University (No. 2021-023). Informed written consent was provided by all patients before inclusion in this study.

Open Access Statement: This is an Open Access article distributed in accordance with the Creative Commons Attribution-NonCommercial-NoDerivs 4.0 International License (CC BY-NC-ND 4.0), which permits the noncommercial replication and distribution of the article with the strict proviso that no changes or edits are made and the original work is properly cited (including links to both the formal publication through the relevant DOI and the license). 
See: https://creativecommons.org/licenses/by-nc-nd/4.0/.

\section{References}

1. Weitz J, Koch M, Debus J, et al. Colorectal cancer. Lancet 2005;365:153-65.

2. Engstrand J, Nilsson H, Strömberg C, et al. Colorectal cancer liver metastases - a population-based study on incidence, management and survival. BMC Cancer 2018;18:78.

3. Bhullar DS, Barriuso J, Mullamitha S, et al. Biomarker concordance between primary colorectal cancer and its metastases. EBioMedicine2019;40:363-74.

4. van Huizen NA, Coebergh van den Braak RRJ, Doukas M, et al. Up-regulation of collagen proteins in colorectal liver metastasis compared with normal liver tissue. J Biol Chem 2019;294:281-9.

5. Alves JM, Prado-López S, Cameselle-Teijeiro JM, et al. Rapid evolution and biogeographic spread in a colorectal cancer. Nat Commun2019;10:5139.

6. Haratani K, Hayashi H, Tanaka T, et al. Tumor immune microenvironment and nivolumab efficacy in EGFR mutation-positive non-small-cell lung cancer based on T790M status after disease progression during EGFRTKI treatment. Ann Oncol 2017;28:1532-9.

7. Lusk CM, Watza D, Dyson G, et al. Profiling the Mutational Landscape in Known Driver Genes and Novel Genes in African American Non-Small Cell Lung Cancer Patients. Clin Cancer Res 2019;25:4300-8.

8. Boutin AT, Liao WT, Wang M, et al. Oncogenic Kras drives invasion and maintains metastases in colorectal cancer. Genes Dev 2017;31:370-82.

9. Yang J, Lin Y, Huang Y, et al. Genome landscapes of rectal cancer before and after preoperative chemoradiotherapy. Theranostics2019;9:6856-66.

10. Li H, Durbin R. Fast and accurate long-read alignment with Burrows-Wheeler transform. Bioinformatics 2010;26:589-95.

11. Cibulskis K, Lawrence MS, Carter SL, et al. Sensitive detection of somatic point mutations in impure and heterogeneous cancer samples. Nat Biotechnol2013;31:213-9.

12. Kanehisa M, Goto S. KEGG: kyoto encyclopedia of genes and genomes. Nucleic Acids Res 2000;28:27-30.

13. Ashburner M, Ball CA, Blake JA, et al. Gene ontology: tool for the unification of biology. The Gene Ontology Consortium. Nat Genet 2000;25:25-9.

14. Weaver B, Wuensch KL. SPSS and SAS programs for comparing Pearson correlations and OLS regression coefficients. Behav Res Methods 2013;45:880-95.

15. Dees ND, Zhang Q, Kandoth C, et al. MuSiC: identifying mutational significance in cancer genomes. Genome Res 2012;22:1589-98.

16. Birkbak NJ, McGranahan N. Cancer Genome Evolutionary Trajectories in Metastasis. Cancer Cell 2020;37:8-19.

17. Berglund E, Maaskola J, Schultz N, et al. Spatial maps of prostate cancer transcriptomes reveal an unexplored landscape of heterogeneity. Nat Commun2018;9:2419.

18. Nebbioso A, Tambaro FP, Dell'Aversana C, et al. Cancer epigenetics: Moving forward. PLoS Genet 2018;14:e1007362.

19. Álvarez-Chaver P, De Chiara L, Martínez-Zorzano VS. Proteomic Profiling for Colorectal Cancer Biomarker Discovery. Methods Mol Biol 2018;1765:241-69.

20. Kumar A, Misra BB. Challenges and Opportunities in Cancer Metabolomics. Proteomics 2019;19:e1900042.

21. Vitale I, Manic G, Coussens LM, et al. Macrophages and Metabolism in the Tumor Microenvironment. Cell Metab 2019;30:36-50.

22. Neidhardt J, Fehr S, Kutsche M, et al. Tenascin-N: characterization of a novel member of the tenascin family that mediates neurite repulsion from hippocampal explants. Mol Cell Neurosci2003;23:193-209.

23. Chiovaro F, Martina E, Bottos A, et al. Transcriptional regulation of tenascin- $W$ by TGF-beta signaling in the bone metastatic niche of breast cancer cells. Int J Cancer 2015;137:1842-54.

24. Russell MW, Raeker MO, KorytkowskiKA, et al. Identification, tissue expression and chromosomal localization of human Obscurin-MLCK, a member of the titin and Dbl families of myosin light chain kinases. Gene 2002;282:237-46.

25. Carlsson L, Yu JG, Thornell LE. New aspects of obscurin in human striated muscles. Histochem Cell Biol 2008;130:91-103.

(English Language Editor: J. Jones)

Cite this article as: Yi H, Liao ZW, Chen JJ, Shi XY, Chen GL, Wu GT, Zhou DY, Zhou GQ, Huang JY, Lian L, Yu ZY, He SB. Genome variation in colorectal cancer patient with liver metastasis measured by whole-exome sequencing. J Gastrointest Oncol 2021;12(2):507-515. doi: 10.21037/jgo-21-9 


\begin{tabular}{|c|c|c|c|c|c|c|c|c|c|c|c|c|}
\hline$\widehat{\varrho}$ & & & & & & & & & & $\begin{array}{l}\text { Table S2 The } \\
\text { deletions (InDe }\end{array}$ & $\begin{array}{l}\text { leotide variant } \\
\text { of all samples w }\end{array}$ & $\begin{array}{l}\text { V) and insertions/ } \\
\text { unted }\end{array}$ \\
\hline $10^{x}$ & & & & & & & & & & Sample name & SNV number & InDel number \\
\hline 8 & $\infty$ & g & مٌ & స్ & ن & $\dot{d}$ & $\int^{2}$ & లె & i & CRC01CvsLM & 1,094 & 326 \\
\hline & & & & & & & & & & CRC01CvsT & 168 & 9 \\
\hline & & & & & & & & & & CRC02CvsT & 303 & 19 \\
\hline & & & & & & & & & & CRC02CvsLM & 300 & 17 \\
\hline 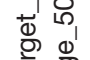 & $\begin{array}{lll}0 & 0 \\
& \stackrel{0}{*}\end{array}$ & $\stackrel{\grave{N}}{\stackrel{N}{N}}$ & $\underset{\dot{x}}{\check{F}}$ & న్ & ז. & ले & $\stackrel{n}{\leftarrow}$ & $\stackrel{\stackrel{\Im}{*}}{\underset{*}{*}}$ & ช్ & CRC03CvsT & 324 & 12 \\
\hline$\digamma^{\sigma} \frac{\pi}{\omega}$ & & & & & & & & & & CRC03CvsLM & 170 & 8 \\
\hline ¿ & & & & & & & & & & CRC04CvsLM & 266 & 20 \\
\hline ఏ & & & & & & & & & & CRC04CvsT & 5,940 & 18 \\
\hline
\end{tabular}

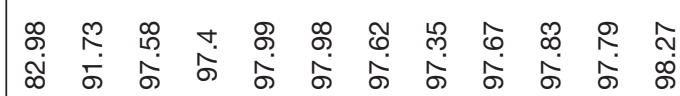

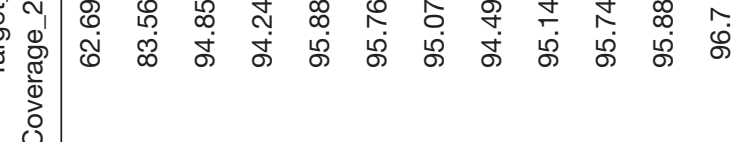
ठำ

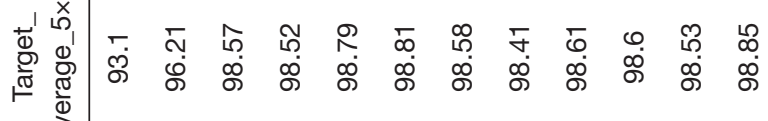

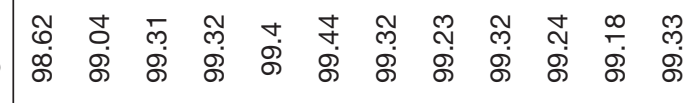
ठे

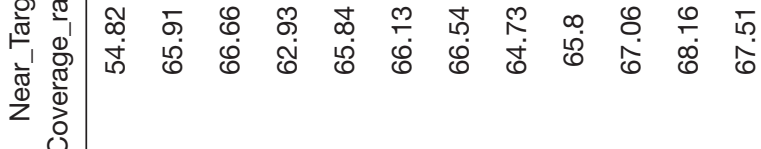
变 $\frac{1}{2}$

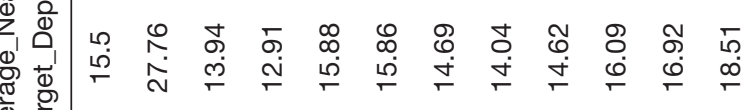
这市

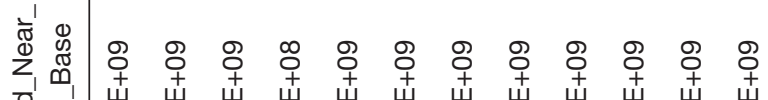

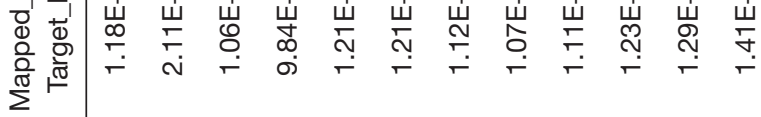

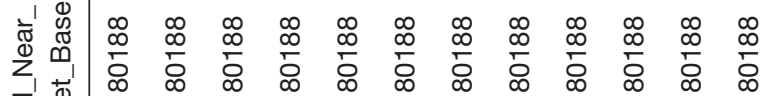
焉兽

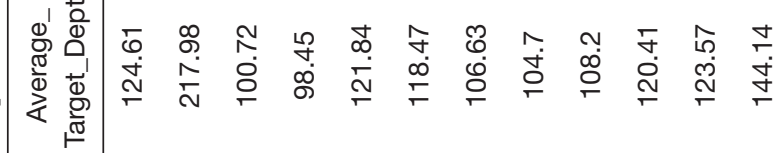
일

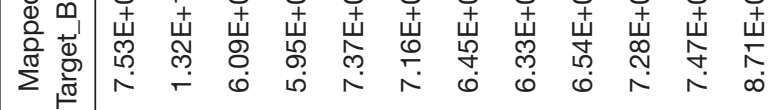

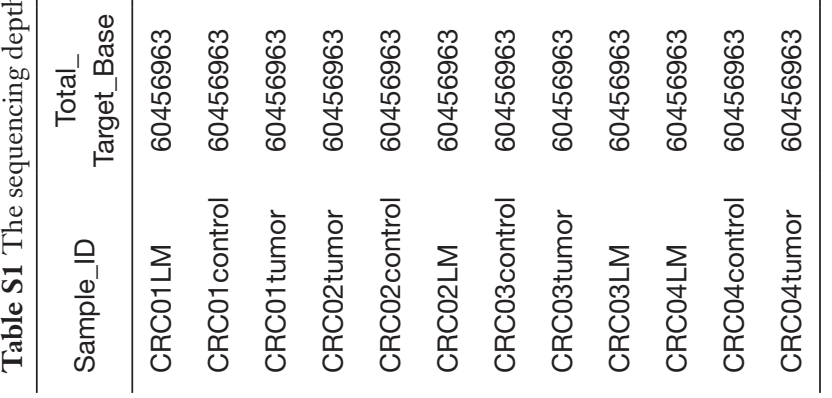

